\title{
Genetic study on emigration behavior of Drosophila melanogaster in a natural population
}

\author{
Kenji Mikasa \\ Department of Biology, School of Dentistry, Meikai University, \\ Sakado, Saitama 350-02
}

(Received 16 June 1990)

\begin{abstract}
Genetic study on emigration behavior of Drosophila melanogaster in the Ishinomaki population was conducted with 140 2nd chromosome lines. Fourteen sets of $5 \times 5$ partial diallel cross experiments were made to examine the emigration activity of $F_{1}$ progeny. Emigration activity was scored using the method of Sakai et al. (1958). Additive genetic variance was $0.0377 \pm 0.0069$ and dominance variance $0.0076 \pm 0.0032$. The average degree of dominance of mildly deleterious genes for emigration activity in an equilibrium population was $0.069 \pm 0.042$. The estimated degree of dominance at a gene locus affecting emigration activity was 0.067 , which revealed nearly complete dominance for the tendency of heterozygote flies to move from their original place to another. Average degree of dominance of lethal gene for emigration activity was 0.012 .
\end{abstract}

\section{INTRODUCTION}

Sakai et al. (1958) began the study on emigration response behavior of Drosophila melanogaster in the laboratory. With the Sakai system, we can measure the potentiality to what extent the member of a population can move to another place. The movement of the flies measured with the system was defined by Rockwell et al. (1978) as emigration response behavior. Many researchers have studied the behavior using the system from the various viewpoints (Narise, 1962, 1966, 1968, 1969, 1974a, b; Narise and Mikasa, 1984, 1988; Takada, 1959; Tantawy et al., 1975; Rockwell et al., 1978, 1983; Rockwell, 1979; Rockwell and Levine, 1986; Mikasa and Narise, 1979, 1983a, b, 1986, 1989). From these studies three findings were obtained: (1) the measured emigration activity is under genetic control, (2) there are genetic variabilities for emigration response behavior in natural populations of $D$. melanogaster (Narise, 1962; Mikasa and Narise, 1986; Narise and Mikasa, 1988), D. pseudoobscura (Rockwell et al., 1983) and D. busckii (Rockwell and Levine, 1986), and (3) genotype-environmental interaction is also an important factor for the emigration response behavior (Narise, 1962; Mikasa and Narise, 1986). So far, it is not known the relationship between emigration activity and dispersal in the natural condition.

A natural population of an organism maintains a large amount of genetic variation. The maintenance of the variation is the fundamental premise of 
evolution, and evolution is caused by the change of genetic variation. Dobzhansky (1955) proposed two predictions for the maintenance of genetic variation in a population: "classical" and "balance" hypotheses. According to Mukai (1978), the "classical" hypothesis states that the genetic variation in a natural population is maintained by the balance of mutation and selection pressure. On the other hand, the "balance" hypothesis proposes that the genetic variability is maintained by the balance of two selection pressures operating in opposite directions. This selection is called "balancing" selection; (1) overdominance, (2) frequency dependent selection, (3) diversifying selection due to spatial or temporal environmental heterogeneity, and so on. However, it is debatable whether or not overdominance is a valid mechanism for the maintenance of genetic variability (Lewontin, 1974; Mukai, 1978).

I, therefore, examined whether or not overdominance is related to the gene affecting the emigration activity of $D$. melanogaster in a natural population. First, fourteen sets of partial diallel cross experiments were conducted. The emigration activities of $F_{1}$ flies in each cross were examined and the analysis of variance on the activities was made to partition the genetic variance components into the additive genetic variance and the dominance variance for the activity. Secondly, the average degree of dominance of mildly deleterious genes for emigration activity in an equilibrium population at the chromosome level was estimated. In addition, the effect of lethal genes on emigration activity was also investigated. Then, I discuss whether or not overdominance is detected at a gene locus affecting emigration activity.

\section{MATERIALS AND METHODS}

\section{Extraction of the second chromosome}

D. melanogaster was collected in September 1983 at Ishinomaki City $\left(\mathrm{N} 38^{\circ} 20^{\prime}\right.$, E141 $\left.11^{\prime}\right)$. Flies were collected from banana-baited traps which were set near houses. Then, 140 isofemale lines were established and maintained at $19^{\circ} \mathrm{C}$.

To examine the effect of only the second chromosome for emigration activity, following procedures were performed. The multiple inversion stock with the markers $C y / P m \quad\left\{\operatorname{In}(2 L R) S M 1 / \operatorname{In}(2 L R) b w^{V 1}\right\}$ was used to extract the second chromosome. The 1st, 3rd, and 4th chromosomes of the stock were made homozygous with an isogenic line of the Ishinomaki population previously. After three generations from the time of capture of the flies, a single male fly of each isofemale line was mated to five virgin females of the multiple inversion stock. Then, a single $\mathrm{F}_{1}$ male progeny with the marker $C y /+$ was backcrossed to the five virgin females of the balancer stock. Subsequently, five male progenies with the marker $\mathrm{Cy} /+$ were backcrossed to the five virgin females of the balancer stock successively for five generations in order to make their genetic backgrounds same. After that period of time five female and five male progenies with the marker 
$\mathrm{Cy} /+$ were mated in each line. These procedures were conducted at $25^{\circ} \mathrm{C}$. Then, the $140 \mathrm{Cy}$ balanced lines were maintained at $19^{\circ} \mathrm{C}$.

\section{Measurement of emigration activity}

Five second chromosome lines were taken for female parents and another five for male parents in order to constitute a $5 \times 5$ partial diallel cross experiment. These lines were selected randomly but without repeat. About fifty virgin females from the $i$ th female line were mated to about fifty males from the $j$ th male line for the $(i, j)$ th cell in a half-pint bottle. These flies were transferred every other day into a new bottle to obtain replicate cultures of each cross at $25^{\circ} \mathrm{C}$. Emigration activity per 100 (50 females and 50 males) $\mathrm{F}_{1}$ wild type flies was scored with the method of Sakai et al. (1958). Detailed procedures for measurement of emigration activity were given earlier (Mikasa and Narise, 1979, 1986). Four replicates were done for each cross at $25 \pm 1^{\circ} \mathrm{C}$ and $60 \%$ relative humidity in random fashion. These experiments were repeated 14 times. There was no missing cell in any set.

\section{Partition of genetic variance}

In a partial diallel cross design, the emigration activity of the $k$ th replicate in a cross of the $i$ th female and the $j$ th male, $Y_{i j k}$, is

$$
Y_{i j k}=\mu+F_{i}+M_{j}+(F M)_{i j}+E_{i j k}
$$

where $\mu$ is the population mean, $F_{i}$ is the effect of the $i$ th female to the progeny, $M_{j}$ the effect of the $j$ th male, $(F M)_{i j}$ the effect of interaction between the $i$ th female and the $j$ th male, and $E_{i j k}$ is the environmental effect on the $k$ th replicate of the progeny of the $i$ th female and $j$ th male. $F_{i}, M_{j},(F M)_{i j}$, and $E_{i j k}$ are all assumed to be random effects, which are normally distributed with a mean of zero and variances $\sigma_{F}^{2}, \sigma_{M}^{2}, \sigma_{F M}^{2}$, and $\sigma_{E}^{2}$, respectively. Based on expression (1), the expected mean squares for the between females $\left(\mathrm{EMS}_{F}\right)$, the between males $\left(\mathrm{EMS}_{M}\right)$, or the interaction between female and male $\left(\mathrm{EMS}_{F M}\right)$ is

$$
\begin{aligned}
& \mathrm{EMS}_{F}=\sigma_{E}^{2}+n_{r} \times \sigma_{F M}^{2}+n_{o} \times n_{r} \times \sigma_{F}^{2} \\
& \mathrm{EMS}_{M}=\sigma_{E}^{2}+n_{r} \times \sigma_{F M}^{2}+n_{o} \times n_{r} \times \sigma_{M}^{2} \\
& \mathrm{EMS}_{F M}=\sigma_{E}^{2}+n_{r} \times \sigma_{M}^{2},
\end{aligned}
$$

respectively, where $\sigma_{E}^{2}$ is the within-cross variance (due to environmental effects); $\sigma_{F}^{2}$ or $\sigma_{M}^{2}$ is the variance component between females or between males, respectively; $\sigma_{F M}^{2}$ is the interaction component; $n_{r}$ is the number of replicates for each cross; and $n_{o}$ is the number of female or male lines used. The variance component $\left(\sigma_{F}^{2}, \sigma_{M}^{2}\right)$ has the additive effect; and the interaction component $\left(\sigma_{F M}^{2}\right)$ indicates the nonadditive one. Thus, the additive genetic variance at the chromosome level $\left(\sigma_{A}^{2}\right)$ is given as the sum of $\sigma_{F}^{2}$ and $\sigma_{M}^{2}$; and dominance variance at the 
chromosome level $\left(\sigma_{D}^{2}\right)$, as the $\sigma_{F M}^{2}$. Before making an analysis of variance, emigration activities of each set were standardized with the mean of emigration activity in each set.

\section{Estimation of average degree of dominance}

Eighteen lines out of the $140 \mathrm{Cy}$-balanced lines used in the diallel cross experiment had semi-lethal genes or were subvital, 45 lines were carrying completely lethal genes in the chromosomes, and the other 77 lines were categorized as having only mildly deleterious genes. Then, the average degree of dominance of mildly deleterious genes or lethal ones for emigration activity was estimated separately.

(1) Mildly deleterious genes for emigration activity. Narise (1974a) reported that there was a significant positive correlation between emigration activity and a component of fitness, duration of egg laying, in inbred lines. Mikasa and Narise (1989) also demonstrated that there was a significant positive correlation between emigration activity and a component of fitness, productivity. Then, if emigration activity is proportional to fitness, the average degree of dominance of genes for emigration activity in an equilibrium population $\left(h_{E}\right)$ can be approximately estimated as follows (Mukai et al., 1972; Mukai and Yamaguchi, 1974):

$$
h_{E} \simeq \beta_{Y \cdot X}=\operatorname{Cov}(X, Y) / \mathrm{V}(X)
$$

where $\beta_{Y \cdot X}$ is the expected regression coefficient of the emigration activity of a heterozygote $(Y)$ on the sum of the emigration activities in the two constituent homozygotes $(X), \mathrm{V}(X)$ is the genetic variance of $X$, and $\operatorname{Cov}(X, Y)$ is the genetic covariance between $X$ and $Y$. The genetic variance and the genetic covariance were estimated from the analysis of variance and from the analysis of covariance. The emigration activities of the 77 lines having mildly deleterious genes were scored using the Sakai system per 100 (50 females and 50 males) wild type flies in homozygote with four replicates for each line. The activity was standardized with the mean emigration activity in the constituting each diallel cross set. The emigration activities of heterozygotes corresponding to the two constituent homozygotes were derived from the data in the diallel cross studies. As a result, 107 crosses were obtained for the analysis.

(2) Lethal genes for emigration activity. 45 lines had lethal genes in their chromosomes. The other lines were grouped as non-lethal chromosome lines for the sake of convenience. The cross experiments offered 350 heterozygotes: 158 lethal-free, 159 single-lethal, and 33 double-lethal.

\section{RESULTS}

Genetic variance

Analysis of variance was conducted for the data in each set. The degrees of 
freedom and sums of squares in each set were pooled to estimate the components of genetic variance at the population level. The pooled results are presented in Table 1 . The variances due to females and males were statistically highly significant $(P<0.001)$, and that due to interaction of female with male was also statistically highly significant $(P<0.01)$. Then, from equations $(2)-(4)$, the components of genetic variances were estimated as follows: $\hat{\sigma}_{A}^{2}=0.0377 \pm 0.0069$ and $\hat{\sigma}_{D}^{2}=0.0076 \pm 0.0032$. Their standard errors were estimated following the method presented in Mukai (1978).

Table 1. Pooled results of analysis of variance on emigration activity

\begin{tabular}{|c|c|c|c|}
\hline Source & d.f. ${ }^{1)}$ & S.S. $^{2)}$ & M.S. ${ }^{3)}$ \\
\hline Total & 1386 & 188.6396 & \\
\hline Females (F) & 56 & 30.6675 & $0.5476^{* * *}$ \\
\hline Males (M) & 56 & 25.9192 & $0.4628^{* * *}$ \\
\hline $\mathrm{F} \times \mathrm{M}$ & 224 & 28.8292 & $0.1287^{* *}$ \\
\hline Error & 1050 & 103.2236 & 0.0983 \\
\hline
\end{tabular}

1) d.f. stands for degree of freedom.

2) S.S. stands for sum of squares.

3) M.S. stands for mean of squares.

** Significant at 0.01 .

*** Significant at 0.001 .

Average degree of dominance of mildly deleterious genes for emigration activity

The relationship between the means of emigration activity in heterozygotes $(Y)$ and the means of the sum of these in the two constituent homozygotes $(X)$ is shown in Fig. 1. The regression coefficient of $Y$ on $X$ was $0.062 \pm 0.038$ and was not statistically different from zero $\left(0.05<P<0.10, t_{105}=1.749\right)$. Further analysis was performed to know whether or not the degree of dominance of gene for emigration activity becomes negative. The negative value of the degree of dominance reveals that overdominance is related to the emigration activity. The genetic variance of $X$ was $\hat{\sigma}_{X}^{2}=0.3457$, and the genetic covariance of $X$ with $Y$ was $\hat{\sigma}_{X Y}^{2}=0.0237$. Then, the average degree of dominance of genes for emigration activity in an equilibrium population $\left(\bar{h}_{E}\right)$ was $\bar{h}_{E}=0.069 \pm 0.042$. The standard error was calculated following the method of Tallis (1959).

\section{Mean of degree of reduction in emigration activity per lethal gene}

Means and standard errors of standardized emigration activity in the lethalfree, single-lethal, and double-lethal heterozygotes are presented in Table 2. There were no significant differences among the means in these groups $(P>0.05$, $F_{2,347}=0.53$ ). Although the lethal genes did not affect emigration activity signif- 


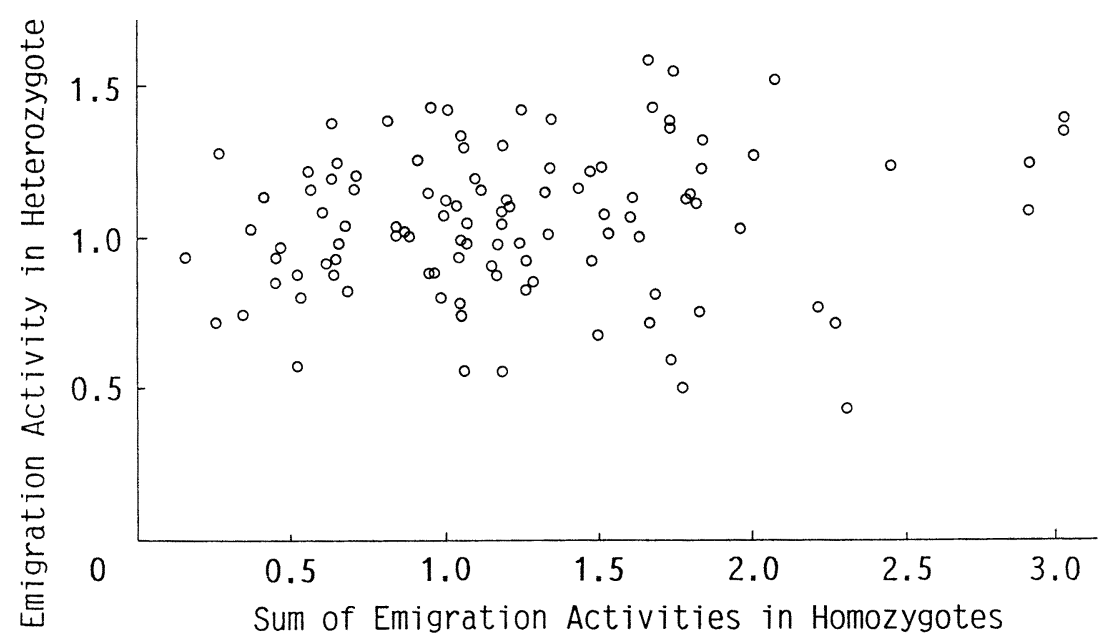

Fig. 1. The relationship between emigration activity in heterozygote and the sum of the activities in the two constituent homozygotes.

Table 2. Means and standard errors of emigration activity in lethalfree, single-lethal, and double-lethal heterozygotes

\begin{tabular}{lcc}
\hline Genotype & $\mathrm{N}^{1)}$ & Emigration Activity \\
\hline Non-lethal/Non-lethal & 158 & $\begin{array}{l}1.009 \pm 0.388 \\
(1.000)^{2)}\end{array}$ \\
Non-lethal/Lethal & 159 & $\begin{array}{l}0.999 \pm 0.350 \\
(0.990)\end{array}$ \\
Lethal/Lethal & 33 & $0.961 \pm 0.326$ \\
& & $(0.952)$ \\
\hline
\end{tabular}

1) $\mathrm{N}$ stands for the number of heterozygotes.

2) The figures in parentheses are standardized with the mean of lethal-free heterozygote.

icantly, an increase in the number of lethal genes seemed to decrease the activity. So, the degree of reduction of emigration activity per lethal gene was estimated following the procedure as mentioned by Mukai (1978). The mean of degree of reduction in emigration activity per lethal gene locus $\left(\hat{\bar{h}}_{l a}\right)$ was $\hat{\bar{h}}_{l a}=0.012$.

\section{DISCUSSION}

In the present study, only the second chromosome was used. The use of $C y$ balanced lines makes possible evaluation of the effects of lethal, semi-lethal, and subvital genes, of which proportion was $45 \%$ in the Ishinomaki population.

From the experimental results it can be said that the majority of genes affecting emigration activity operates additively, although a small effect of dominance is detected. 
As the effect of an individual locus for emigration activity is considered to be small, it is impossible to estimate the degree of dominance for emigration activity at a locus level. It is assumed that the mutant genes of emigration activity are distributed at random on the chromosome and there was no linkage disequilibrium between loci. In addition to these, there are $n$ loci on the chromosome with two alleles and there is additive effect among loci. The regression coefficient at the chromosome level, $\beta_{Y \cdot X}$ [Equation (5)], is as follows (Mukai and Yamaguchi, 1974):

$$
\beta_{Y \cdot X}=\frac{\sum_{i=1}^{n} 2 p_{i} q_{i}\left[h_{i}+q_{i}\left(1-2 h_{i}\right)\right] s_{i}{ }^{2}}{\sum_{i=1}^{n} 2 p_{i} q_{i} s_{i}{ }^{2}}
$$

where $p_{i}$ or $q_{i}\left(p_{i}+q_{i}=1\right)$ is gene frequency of "normal" or mutant allele at locus $i$ for emigration activity respectively, $h_{i}$ is the degree of dominance of the mutant allele at the locus $i$, and $s_{i}$ is the selection coefficient for the mutant homozygote at the locus $i$.

If all loci have the same effect on emigration activity, the frequency of mutant gene, the degree of dominance of gene affecting emigration activity, and selection coefficient would be the same values among all loci respectively. Then, $\beta_{Y \cdot X}$ is simplified:

$$
\beta_{Y \cdot X} \simeq h+q(1-2 h) .
$$

$\beta_{Y \cdot X}=h_{E}$ [equation (5)] and the obtained estimate is $\bar{h}_{E}=0.069$. Then, the degree of dominance of gene affecting emigration activity is

$$
h \simeq(0.069-q) /(1-2 q) .
$$

In one locus with two alleles, the additive genetic variance $\left(\sigma_{a}^{2}\right)$ and dominance variance $\left(\sigma_{d}^{2}\right)$ are as follows (Mukai et al., 1974):

$$
\begin{aligned}
\sigma_{a}^{2} & =2 p q[(p-q) h+q]^{2} s^{2} \\
\sigma_{d}^{2} & =p^{2} q^{2}(1-2 h)^{2} s^{2} .
\end{aligned}
$$

The ratio of additive genetic variance to dominance variance at the chromosome level $(R)$ is

$$
\mathrm{R}=\frac{\sum_{i=1}^{n} 2 p_{i} q_{i}\left[\left(p_{i}-q_{i}\right) h_{i}+q_{i}\right]^{2} s_{i}{ }^{2}}{\sum_{i=1}^{n} p_{i}{ }^{2} q_{i}{ }^{2}\left(1-2 h_{i}\right)^{2} s_{i}{ }^{2}} .
$$

From the same assumption above, the ratio is simplified as follows:

$$
\mathrm{R} \simeq 2[(p-q) h+q]^{2} /\left[p q(1-2 h)^{2}\right] .
$$


The estimated ratio $(\hat{\mathrm{R}})$ was $\hat{\mathrm{R}}=4.96(=0.0377 / 0.0076)$. Then, from equations $(6)$ and (7), and $p+q=1$, two solutions for $q$ were obtained as follows: 0.003 and 0.997 . And from equation (6), $\hat{q}=0.003$ gives $\hat{h}=0.067$ and $\hat{q}=0.997, \hat{h}=0.933$. As $\hat{h}=0.933$ is significantly deviated from the average degree of dominance of genes for emigration activity in an equilibrium population $\left(\bar{h}_{E}=0.069 \pm 0.042\right), \hat{h}=0.933$ is abandoned. Therefore, the degree of dominance in emigration activity is $\hat{h}=0.067$.

Gene frequency of mutant allele at one locus for emigration activity is very small, nearly zero. Two reasons may be expected: 1) There is a strong selection against "staying at the same place", because the flies which can move to another place would gain the possibility for the increase of fitness, and 2) the second chromosome has little effect on emigration behavior, although the effect of the second chromosome is not zero. The obtained degree of dominance shows nearly complete dominance. As the degree of dominance of a gene for emigration activity is neither negative nor larger than 1, overdominance is not related to a gene locus for emigration activity.

The mean of degree of reduction in emigration activity per lethal gene locus is equivalent to the average degree of dominance for emigration activity at a lethal gene locus. The value is $\hat{\bar{h}}_{l a}=0.012$. However, lethal gene had little effect to emigration activity.

In conclusion, most of genetic variance components for emigration activity was the additive genetic variance, although a small portion of dominance variance was detected. Overdominance is not related to the mechanism of the maintenance for genetic variation of emigration activity in a natural population of $D$. melanogaster.

I thank the late Prof. T. Mukai, Kyushu University, for his valuable suggestions with respect to this study and his critical reading of this manuscript. I am grateful to Prof. T. Narise, Meikai University, for his critical reading of the manuscript. I thank Prof. H. Matsuda and Dr. T. Yamazaki, Kyushu University, for their valuable comments on this study. I am also grateful to an anonymous referee for his valuable comments. I thank Dr. L. D. Frye for his corrections of the English of the manuscript.

\section{REFERENCES}

DobZhansky, Th. (1955) A review of some fundamental concepts and problems of population genetics. Cold Spring Harbor Symp. Quant. Biol. 20, 1-15.

Johnson, C. G. (1969) Migration and Dispersal of Insects by Flight, Metheun, London.

Lewontin, R. C. (1974) The Genetic Basis of Evolutionary Change, Columbia Univ. Press, New York.

MIKASA, K. and NARISE, T. (1979) The relation between dispersive behavior and temperature in Drosophila melanogaster. I. Dispersal pattern. Jpn. J. Genet. 54, 217-228.

MiKASA, K. and NARISE, T. (1983a) Interactive effects of temperature and geography on emigration behavior of Drosophila melanogaster: Climatic and island factors. Behav. Genet. 13, 29-41.

MiKASA, K. and NARISE, T. (1983b) Interactive effects of temperature and geography on emigration behavior of Drosophila melanogaster. II. Further studies on geographical differences. Jpn. J. Genet. 58, 487-496. 
MiKASA, K. and NARISE, T. (1986) Genetic variation of temperature-influenced emigration behavior of Drosophila melanogaster in a natural population. Jpn. J. Genet. 61, 233-240.

MIKASA, K. and NARISE, T. (1989) Interactive effects of temperature and geography on emigration behavior and productivity of Drosophila melanogaster in northern and western Japan. J. Arts Sci. Meikai Univ. 1, 1-13.

MukaI, T. (1978) Population Genetics. Kodansha Scientific, Tokyo. (in Japanese).

Mukai, T., Cardellino, R. A., Watanabe, T. K. and Crow, J. F. (1974) The genetic variance for viability and its components in a local population of Drosophila melanogaster. Genetics 78, 11951208.

Mukai, T., Chigusa, S. I., Mettler, L. E. and Crow, J. F. (1972) Mutation rate and dominance of genes affecting viability in Drosophila melanogaster. Genetics 72, 335-355.

MukAI, T. and YAMAGUCHI, O. (1974) The genetic structure of natural populations of Drosophila melanogaster. XI. Genetic variability in a local population. Genetics 76, 339-366.

NARISE, T. (1962) Studies on competition in plants and animals X. Genetic variability of migratory activity in natural population of Drosophila melanogaster. Jpn. J. Genet. 37, 451-461.

NARISE, T. (1966) The mode of migration of Drosophila ananassae under competitive condition. Univ. Texas Publ. 6615, 121-130.

NARISE, T. (1968) Migration and competition in Drosophila I. Competition between wild and vestigial strains of Drosophila melanogaster in a cage and migration-tube population. Evolution 22, 301-306.

NARISE, T. (1969) Migration and competition in Drosophila II. Effect of genetic background on migratory behavior of Drosophila melanogaster. Jpn. J. Genet. 44, 297-302.

NARISE, T. (1974a) Relation between dispersive behavior and fitness. Jpn. J. Genet. 49, 131-138.

NARISE, T. (1974b) Genetics and evolution of emigration behavior. In: Behavior and Adaptation in Insects, (ed.: C. Oshima), Baifukan, Tokyo. (in Japanese).

NARISE, T. and MIKASA, K. (1984) The effect of another population on emigration behavior of a population. Jpn. J. Genet. 59, 51-59.

NARISE, T. and MIKASA, K. (1988) Genetic variation of emigration behavior in natural populations of Drosophila melanogaster. Genet. (Lif. Sci. Adv.) 7, 141-143.

RockWELl, R. F. (1979) Emigration response behavior: II. The responses of Drosophila busckii. Pan-Pac. Entomol. 55, 117-126.

Rockwell, R. F., Grossfield, J. and Levine, L. (1978) Emigration response behavior: I. Effects of height and light on the Oregon-R and norp-A strains of Drosophila melanogaster. Egypt. J. Genet. Cytol. 7, 123-136.

Rockwell, R. F. and Levine, L. (1986) Emigration response behavior: III. Genetic variability in a natural population of Drosophila busckii. Behav. Genet. 16, 543-551.

Rockwell, R. F., de la Rosa, M. E., Akin, E., Gaso, M. I., Gonalez, F., Guzman, J., Levine, L. and OlverA, O. (1983) Chromosomal and behavioral studies of Mexican Drosophila. I. Vagility characteristics of three populations of D. pseudoobscura. Behav. Genet. 13, 197-204.

Sakai, K., NARISE, T., Hiraizumi, Y. and IYAMA, S. (1958) Studies on competition in plants and animals. IX. Experimental studies on migration in Drosophila melanogaster. Evolution 12, 93-101.

Takada, H. (1959) Migration of some Drosophila in population tubes. Dros. Inf. Serv. 33, 167.

TALlis, G. M. (1959) Sampling errors of genetic correlation coefficients calculated from analysis of variance and covariance. Aust. J. Stat. 1, 35-43.

Tantawy, A. O., Mourad, A. M. and Abou-Youssef, A. A. (1975) Studies on natural populations of Drosophila. XVI. Migration in Drosophila melanogaster in relation to genotype, temperature and population density. Egypt. J. Genet. Cytol. 4, 263-276. 